\title{
Moldeo subacuático de objetos arqueológicos
}

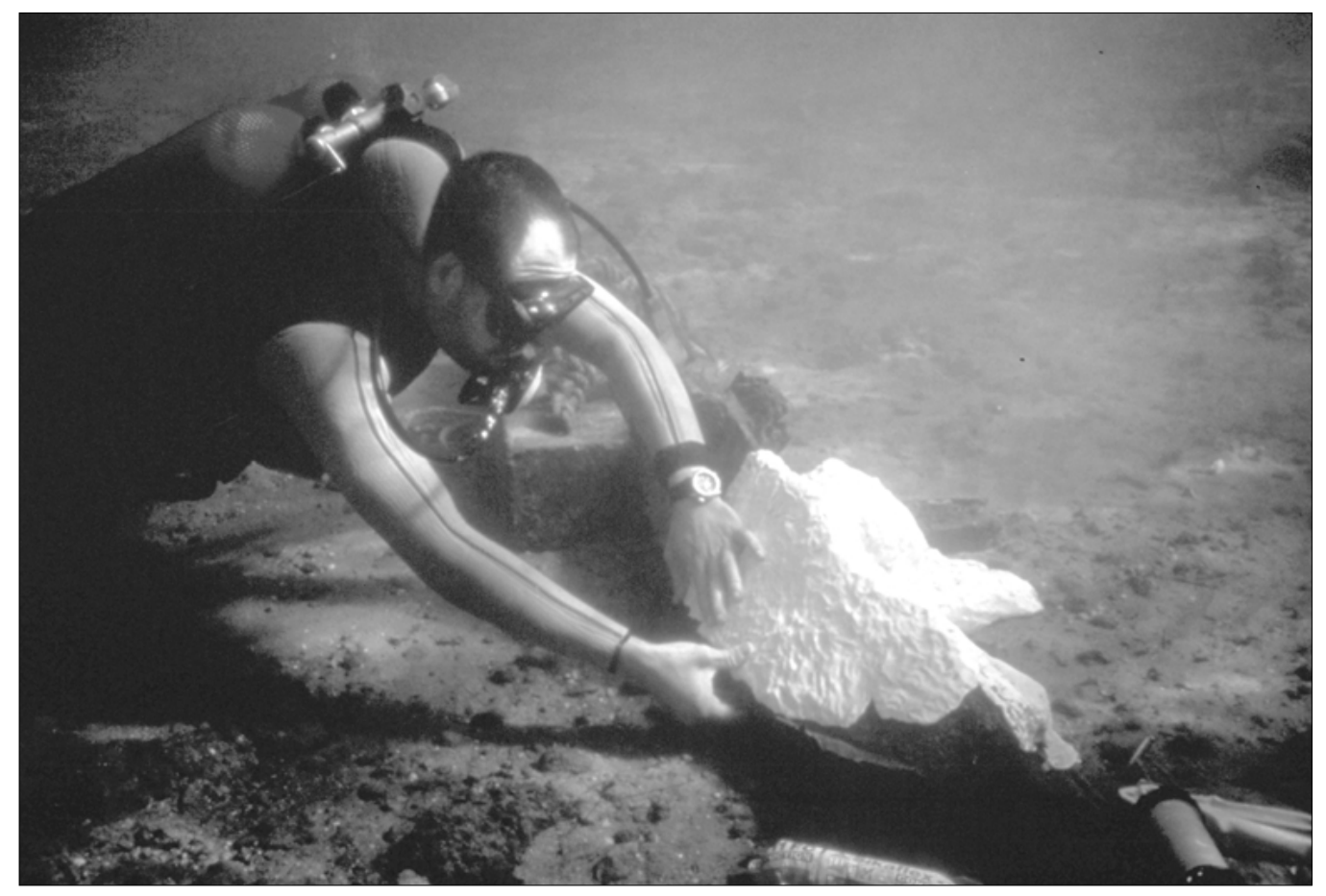

\section{Luis Carlos Zambrano Valdivia}

Centro de Arqueología Subacuática del IAPH

\section{Resumen}

El desarrollo técnico de las herramientas de estudio y la optimización de los tratamientos de conservación tiene una efecto evidente en el conocimiento y la preservación del Patrimonio Histórico Subacuático. El molde a escala $|:|$ es un registro tridimensional de los objetos sumergidos que complementa eficazmente el trabajo convencional de documentación in situ realizado mediante dibujo y fotografía, pudiendo de este modo completarse un estudio muy preciso de los objetos sin necesidad de ser extraídos. Además, el molde supone un sistema de fijación excelente para la recuperación de objetos muy debilitados con un riesgo potencial de fractura y desmembración durante su traslado a la superficie.

El objeto de este trabajo ha sido el determinar un método eficaz para el moldeo in situ de los restos materiales que constituyen el Patrimonio Arqueológico Subacuático. Como resultado se ofrece un método normalizado del moldeo subacuático aplicable al registro arqueológico, patentado en España con el título "Nuevo Procedimiento para la Obtención de Moldes Subacuáticos".

\section{Palabras clave}

Arqueología subacuática / moldes / elastómero de silicona / resina de poliéster / conservación in situ / registro arqueológico / madera sumergida 


\section{Introducción}

En la investigación de los yacimientos subacuáticos y terrestres se aplica, en esencia, un solo método de conocimiento cientííco, el método arqueológico. Las diferencias del medio no exigen la modificación del método pero sí de la técnica y sus herramientas. La arqueología subacuática precisa de herramientas específicas para su ejecución porque el medio donde se desarrolla dicha actividad condiciona todos los elementos que intervienen en el proceso de investigación. Desde las personas que trabajan con el apoyo de sistemas externos para completar su función respiratoria hasta las herramientas diseñadas para desempeñar las funciones imprescindibles de la actividad arqueológica (excavación, dibujo, fotografía, conservación, etc..), toda la infraestructura responde a las características de un medio adverso. Sin embargo, este medio cada vez resulta mas accesible debido a los adelantos técnicos que la arqueología aprovecha para optimizar sus métodos de investigación y análisis. Así, se ha avanzado considerablemente en el conocimiento histórico de dos tipos de yacimientos: los fondeaderos y los barcos '. Estos yacimientos aportan un gran volumen de información sobre aspectos poco conocidos de la actividad humana relacionada con el mar. Aunque por otra parte, también presenten problemas de alteración muy específicos que todavía no están completamente solucionados como es la conservación de las maderas empapadas en agua.

Actualmente, el patrimonio histórico se considera objeto tanto de investigación como de conservación. La relación entre ambos fines ha sido desigual y como consecuencia muchos restos arqueológicos solo perviven en los anales de la arqueología a través de los datos recogidos durante su excavación. Esta realidad, camino de ser completamente superada, ha privado a los investigadores contemporáneos de analizar con los medios actuales aquellos objetos que ya no existen o están completamente arruinados. En este sentido, la conservación no es un freno a la investigación como puede derivarse de noticias como la moratoria de excavación anunciada por Irlanda en 1988 para los galeones españoles hundidos en sus costas ${ }^{2}$. Al contrario, es una garantía de pervivencia para los objetos del patrimonio mantenidos in situ o extraídos a la superficie que pueden seguir siendo estudiados en el tiempo. En este sentido, muchas instituciones de arqueológica subacuática vienen trabajando en la conservación in situ de restos sumergidos como una alternativa a la extracción de materiales cuya conservación está dificultada por cuestiones de índole técnica y económica ${ }^{3}$. En esta línea están enmarcados los trabajos de investigación en el moldeo subacuático de objetos arqueológicos.

\section{Proceso de trabajo}

Numerosos ensayos ${ }^{4}$ de moldeo subacuático se han realizado con resultados dispares que analizados en conjunto constituyen una experiencia acumulada muy útil para abordar el objetivo propuesto en este trabajo que es la definición de un método normalizado de moldeo subacuático aplicado al registro arqueológico y con utilidad en la extracción de restos arqueológicos fragilizados.

En este trabajo se ha empleado un método experimental consistente en la revisión crítica de los diferentes sistemas de moldeo recogidos en la documentación bibliográfica anexa y la formulación teórica de técnicas potencialmente eficaces. En el primer caso, solo se ha profundizado en los ensayos mas recientes y perfeccionados obviando técnicas ya superadas con escasa garantía de éxito que a priori han sido descartadas. En el segundo caso, se realizaron inicialmente numerosos experimentos con planteamientos técnicos y materiales muy diversos para ir progresivamente definiendo un método mas preciso donde los ensayos solo planteaban mejoras de forma respetando un planteamiento básico aceptado. Ambos procesos se han yuxtapuesto en el tiempo y complementado en el conocimiento. Todos los ensayos han sido objeto de una minuciosa documentación que ahora constituye una notable base de datos experimentales. Finalmente, el resultado de ambas líneas es la proposición de un sistema inédito de moldeo subacuático, registrado con la PATENTE DE INVENCIÓN ES-2 I 19649 que presenta sustanciales ventajas respecto a los sistemas tradicionalmente empleados 5 .

\section{Molde flexible y soporte rígido}

El proceso de moldeo consta de dos fases perfectamente definidas: el moldeo flexible y el soporte rígido. Ambas son complementarias y se hacen en el orden expresado I) molde flexible y 2) soporte rígido. El primero registra o reproduce todos los detalles del objeto. Crea un copia en "negativo" del original. Al ser flexible puede extraerse con facilidad sin alterar el soporte y las copias. Por este cualidad, el molde necesita la participación del soporte rígido para conservar la forma del objeto. El soporte se realiza de un modo independiente sobre la cara no registrada del molde y su función es mejorar los resultados del primero.

Los primeros ensayos destacados para obtener moldes flexibles subacuáticos son obra de L. y T. del Wet Sites Conservation Laboratory ${ }^{6}$. Emplearon un caucho de polisulfuro como agente de moldeo subacuático. Se trata de un elastómero bicomponente que cura en presencia de humedad. En ensayos de laboratorio emplearon el elastómero fluido, aplicado en "colada" sobre modelos de tamaño reducido. plantearon el moldeo de volúmenes exentos (por ejemplo, un cilindro) y espacios huecos (por ejemplo, una grieta). Los registros o reproducciones fueron buenos pero sin control directo en la aplicación del elastómero. El caucho de polisulfuro es un fluido mas denso que el agua y tiende a ocupar los espacios inferiores. En la práctica, el caucho fluido resbala por la superficie del objeto a reproducir. Para retener al fluido es necesario encerrarlo entorno al modelo con algún sistema de contención. La confección de un dique de contención propuesto por Murdock y Daley plantea innumerables proble- 
mas técnicos añadidos. Básicamente se condiciona la viabilidad del molde subacuático a la posibilidad de crear un dique contenedor del producto en estado fluido. El moldeo de una gran superficie (3-4 m2) hace inviable el procedimiento de colada aplicado en el laboratorio por Murdock y Daley. El sistema obliga a rellenar con elastómero el espacio comprendido entre las cotas mayor y menor de la superficie a reproducir. Por este motivo, en superficies muy accidentadas, se llega a un excesivo gasto de material siguiendo dicho procedimiento.

Ejemplo: Para reproducir el casco interior de un barco de 7 metros de eslora total y 1,5 metros de manga en el medio con una diferencia de cotas entre la línea de borda y el ensamble de la quilla de 1,5 metros, tenemos un volumen interior aproximado de 6 metros cúbicos. La superficie a reproducir es de 18 metros cuadrados y se necesitarían por tanto unos 6.000 litros de elastómero. La descripción esquemática de la técnica se puede ver en la imagen $n^{\circ} 2$

Otra solución técnica diferente fue planteada por los mismos Murdock y Daley en 1983 sobre los restos del ballenero vasco San Juan, hundido en 1565 a 10 metros de profundidad frente a la costa de Canadá. Se trata del sistema que se ha denominado "en compresa" debido al procedimiento de aplicación del caucho para el moldeo. Básicamente se trata de embadurnar un trozo de tejido con el elastómero y posteriormente aplicarlo sobre la superficie a reproducir. El operador presiona la compresa de tejido y caucho sobre el modelo obteniendo un mayor control sobre el proceso de moldeo.

El sistema de compresa aumenta notablemente el control de aplicación y reduce el gasto de material pero presenta graves inconvenientes.

I. El caucho resulta muy adherente y el traslado de la compresa hasta el punto de aplicación requiere la utilización de complicados artilugios de transporte como bastidores o bandejas de aprehensión.

2. La adaptación de la compresa al sustrato del modelo no es buena cuando la superficie es accidentada. El tejido carece de plasticidad para adaptarse y el operador no controla visualmente el grosor del molde que aplica.

3. El molde resultante presenta zonas con registro insuficiente, puntos de adelgazamiento o ausencia total de elastómero.

Otras variaciones de los sistemas de colada y compresa han sido empleados por G. Brocot en el Centro de Investigaciones Arqueológicas Submarinas de Francia en 1987. Brocot utiliza elastómeros de silicona RTV -Room Temperature Vulcanization- con los ya conocidos métodos de "colada" y "compresa". Estos ensayos no ofrecen ventajas destacables sobre el sistema original de Murdock y Daley ya que mantienen el planteamiento básico de operatividad con la variante del material de moldeo.

\section{Soporte rígido}

Para la obtención de soportes rígidos subacuáticos se ha venido empleando sistemáticamente la escayola que se prepara en superficie del modo convencional y se transporta bajo el agua hasta el lugar de aplicación. Sin embargo, este material presenta serios inconvenientes que lo hacen desaconsejable.

I. El transporte y manipulación subacuática genera una molesta nube blanquecina de polvo en suspensión que dificulta la visibilidad subacuática ya reducida por la falta de luz.

2. Se aplica en estado fluido con problemas de manipulación similares a los descritos para los productos de moldeo flexible aunque no existe la posibilidad de aplicación en compresa.

3. La escayola fraguada bajo agua no endurece por completo. El producto resultante es blando y escasamente homogéneo.

4. Se precisa un grosor muy elevado en relación a la superficie para garantizar la firmeza del soporte. Por ejemplo, un soporte rígido fabricado en escayola para una superficie de I metro cuadrado precisa un grosor aproximado de 30 a 40 centímetros, lo que supone un gasto de 30 a 40 litros de escayola líquida.

5. El peso de la escayola endurecida junto al gran volumen necesario hacen del soporte rígido de escayola un elemento de costosa manipulación dentro y fuera del agua.

Imagen I
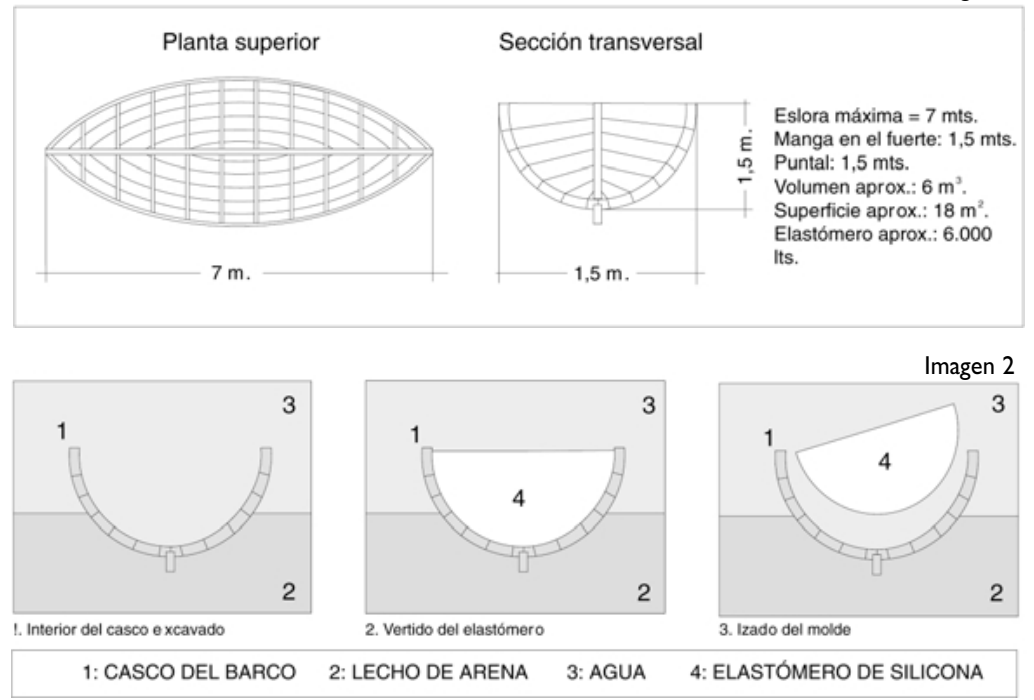

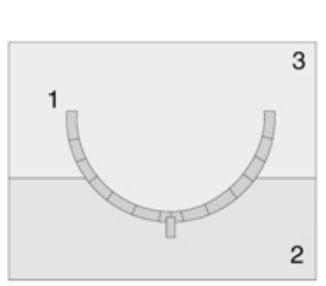

Barco in situ excavado 1: CASCO DEL BARCO

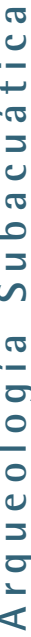

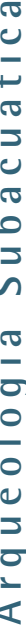

,




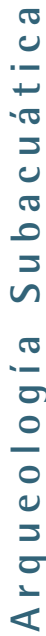

\section{Sistema combinado con silicona y resina de poliéster (2-10)}

Con el fin de mejorar el sistema de obtención de moldes subacuáticos respecto a los sistemas conocidos se ha desarrollado un nuevo procedimiento que presenta notables ventajas sobre los anteriores. La novedad del procedimiento reside en el sistema de aplicación de los productos empleados, elastómero de silicona RTV y resina de poliéster. Así como en la combinación específica de los mismos.
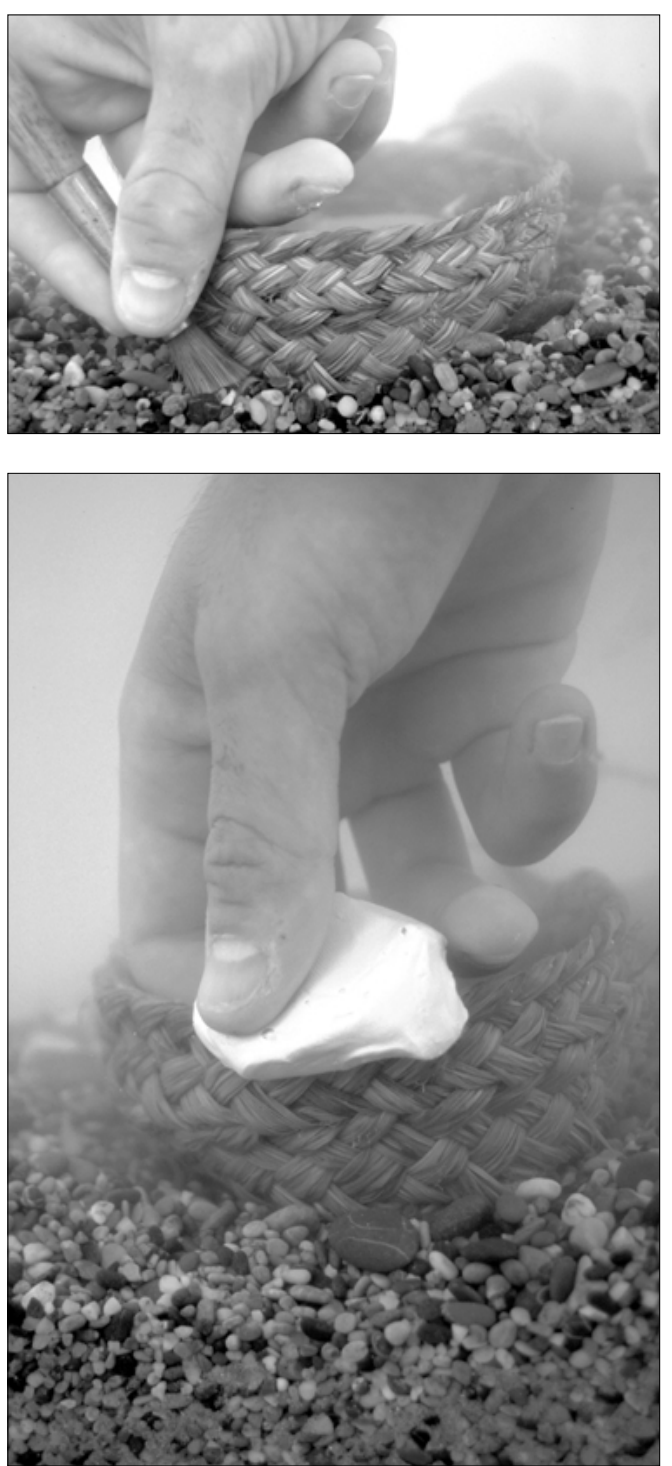

Frente a los anteriores métodos de aplicación en "colada" -producto fluido- o en "compresa" -producto estratificado- aquí se propone un sistema de aplicación controlada que a partir de ahora se llamará en "adición".

Los productos usados para la obtención del molde flexible son: un elastómero de silicona tipo RTV, un catalizador de silicato de etilo como agente de curado y un aditivo tixotrópico convencional. Pueden usarse distintos tipos de catalizadores de silicato de etilo en función del tiempo de curado que se estime adecuado. El tipo "estandar" tiene un tiempo de curado máximo de hasta 24 horas. Hay un catalizador "rápido" con un tiempo de curado entre 3 y 4 horas y un catalizador "muy rápido" efectivo entre I y 2 horas.

Siempre que el tiempo de curado no sea un condicionante inexcusable del trabajo se recomienda usar el agente de curado tipo "estandar" ya que se obtiene mejor calidad en el registro.

\section{Elaboración del molde flexible}

El método de preparación de la masilla para fabricar el molde flexible es el siguiente:

I. Se mezcla la base del elastómero de silicona con el agente de curado en la proporción de 3 a 5 \% en volumen de este respecto de la silicona.

2. Se baten enérgicamente los dos productos durante 3 minutos hasta obtener una mezcla homogénea.

3. Se añade el aditivo tixotrópico en proporción de 10 $\%$ en volumen sobre el volumen inicial de la base de silicona.

4. Se bate enérgicamente la mezcla durante 3 minutos hasta lograr una masa densa que no descuelgue en posición vertical.

La masilla así preparada está lista para su aplicación inmediata. Recién preparada tiene un "tiempo de trabajo óptimo" de 35 minutos aproximadamente para su empleo dentro del agua. Debe evitarse el contacto con el producto fuera del agua por ser muy adherente y difícil de eliminar hasta su completo curado. Todos los productos usados en la mezcla son sensibles a la humedad por lo que deben extremarse el cuidado en evitar el agua ante el riesgo de inhibición.

La aplicación del producto se realiza del siguiente modo:

I. Se introduce el recipiente con la masilla dentro del agua.

2. Se toma con la mano una pella de silicona para aplicarla con una ligera presión sobre la superficie del objeto a reproducir cubriendo toda la extensión deseada.

3. Se espera hasta un máximo de 24 horas para lograr el curado completo de la silicona. 
Deben tenerse en cuenta las siguientes advertencias:

I. La calidad de registro obtenida con la silicona aplicada tras el "tiempo óptimo de trabajo" disminuye proporcionalmente al tiempo de sobrepaso.

2. Superando excesivamente el "tiempo óptimo de trabajo" la silicona deja de ser manipulable.

3. El molde obtenido es tanto mas flexible cuanto menor sea el grosor de la capa de silicona aplicada.

4. La adición manual de silicona permite el ahorro de producto y una capa excesivamente gruesa no mejora la calidad del registro.

5. En zonas complicadas (detalles minuciosos y pequeñas cavidades) debe insistirse con ligeras presiones digitales para lograr una mejor adaptación del producto.

\section{Elaboración del soporte rígido}

El soporte rígido se prepara a base de resina de poliéster modificada con cargas inorgánicas. Se aplica sobre el molde de silicona ya curado adaptándose a la superficie exterior del mismo. Los productos empleados para la obtención de la masilla de poliéster para uso subacuático son: una resina de poliéster tixotrópica y preacelerada como base, silice coloidal pirogenada como carga, y peróxido de metiletilcetona como catalizador de la reacción.

El método de preparación de la masilla de poliéster para la obtención del soporte rígido es el siguiente:

I. Se mezcla la resina de poliéster tixotrópica con silice coloidal en polvo en proporción volumétrica 1:2 ( I vol. de resina $\times 2$ vol. de sílice).

2. Se agita enérgicamente la mezcla hasta obtener una masilla de aspecto untuoso.

3. Se mezcla la masa obtenida con el peróxido de metiletílcetona en proporción de $4 \%$ en volumen de este con respecto a la masa agitando enérgicamente hasta lograr un reparto homogéneo.

La masilla obtenida según este procedimiento permite un rápido fraguado. Puede aplicarse manualmente. Presenta unas buenas condiciones de dureza y consistencia post-curado. Ofrece un buen rendimiento y un bajo coste económico. Es necesario proteger la piel, los ojos y las vías respiratorias cuando se manipulan los componentes de la masilla de poliéster. Recién preparada tiene un 2tiempo óptimo de trabajo" de 15 minutos para su empleo dentro del agua.

La aplicación de la resina de poliéster para el soporte rígido se realiza del siguiente modo:

I. Se introduce el recipiente con la masila dentro del agua.
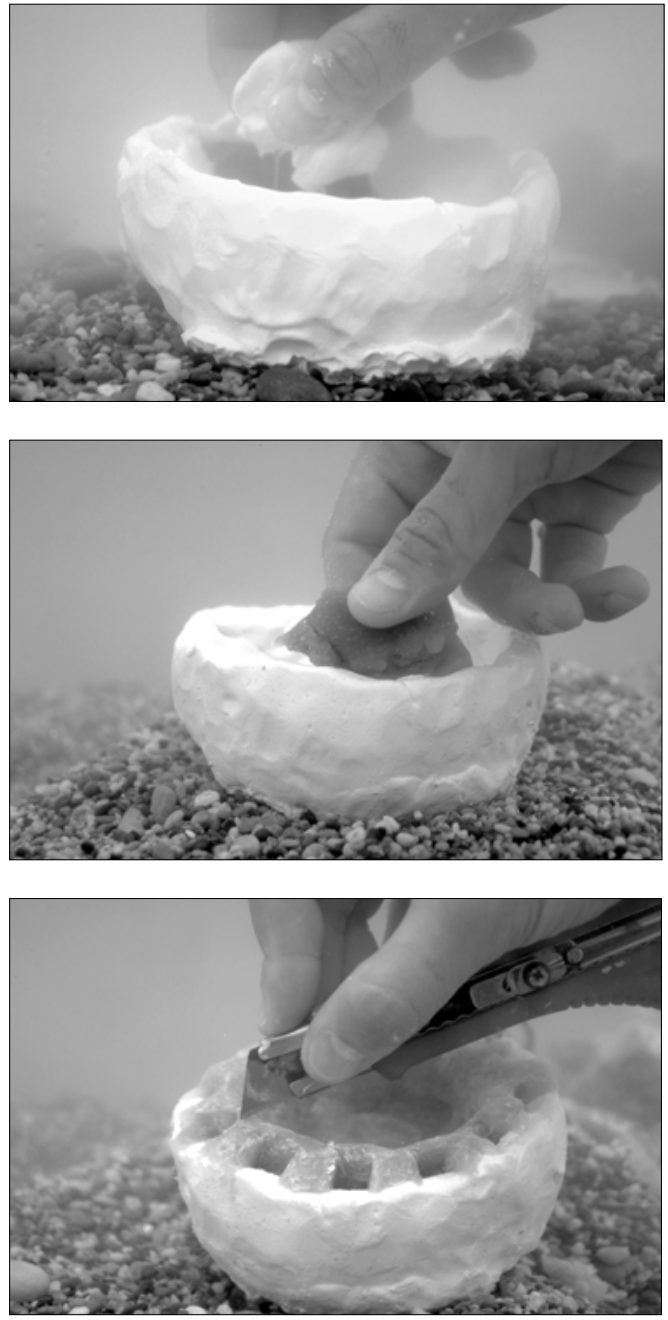

2. Se toma con la mano una pella de masilla para aplicarla manualmente.

3. Se reparte la masilla modelando según las necesidades del trabajo.

4. Se espera hasta un máximo de 15 horas para garantizar un endurecimiento completo del producto. Al cabo de I hora ha adquirido aproximadamente el $75 \%$ de la consistencia final.

Deben tenerse en cuenta las siguientes advertencias:

I. Si se sobrepasa el "tiempo óptimo de trabajo", I5 minutos, la masilla deja de ser manipulable.

2. La manipulación por encima del tiempo recomendado, 15 minutos, merma las características de resistencia del producto terminado.

3. Los tiempos señalados en la preparación del soporte rígido y el molde flexible son indicativos y aproximados. Se basan en experiencias de laboratorio y campo realizadas en condiciones particulares. Consecuentemente, los tiempos pueden verse afectados por la variación en las condiciones del medio o cualquier alteración sustancial del método de trabajo establecido. 

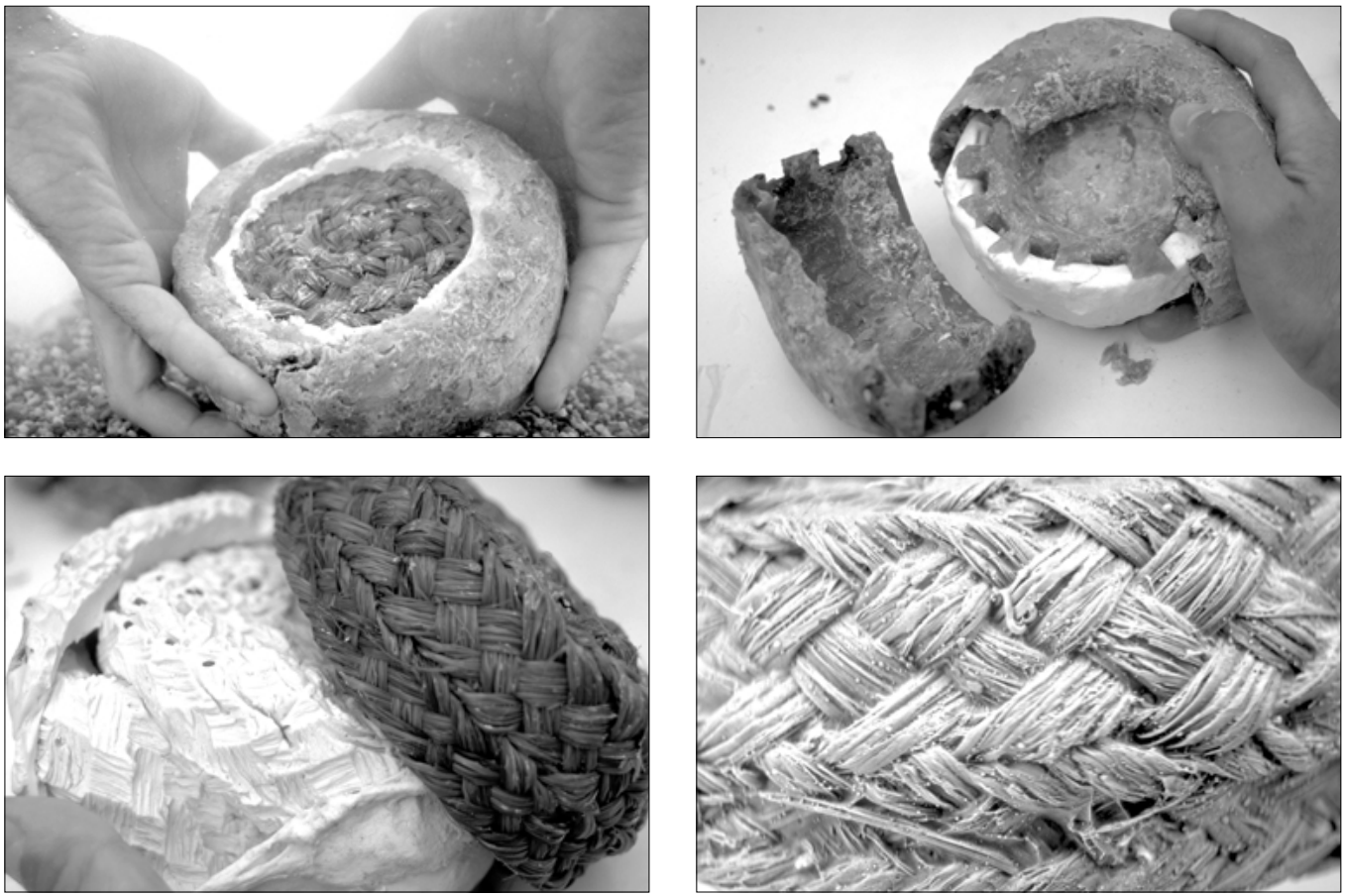

Una vez completados el molde flexible y el soporte rígido se procede a su extracción y posterior montaje. Para la reproducción del objeto moldeado existen infinidad de productos en el mercado. En el caso de la madera es muy aconsejable el empleo de resinas epoxy con cargas celulósicas. En el proceso de reproducción o "positivado" el molde flexible y el soporte rígido forman una sola unidad donde el primero aporta la precisión del detalle y el segundo mantiene la forma del objeto sumergido.

A título meramente ilustrativo, y sin que ello signifique ninguna limitación en el procedimiento, se expone a continuación un ejemplo descriptivo de su ejecución.

La técnica desarrollada permite un control directo en la aplicación del producto, elastómero RTV o resina de poliéster. Esta cualidad del método aporta ventajas interesantes sobre los anteriores procedimientos en: manipulación (gráfico $n^{\circ} \mathrm{I}$ ), adaptación al objeto (gráfico $n^{\circ} 2$ ), desmoldeo (gráfico $n^{\circ} 3$ ), elementos auxiliares (gráfico $n^{\circ} 4$ ), elaboración modular (gráfico $n^{\circ} 5$ ), consumo de producto (gráfico $n^{\circ} 6$ ) y calidad de registro (gráfico nº 7).

\section{Valoración del método}

\section{Ejemplo práctico}

Se describe la preparación subacuática del molde flexible y el soporte rígido del casco interior de un barco sumergido en el mar a 10 metros de profundidad. El barco tiene unas dimensiones de 7 metros de eslora máxima, 1,5 metros de eslora en el fuerte, un puntal de 1,5 metros y un volumen total interior de 6 metros cúbicos con una superficie total a reproducir de 18 metros cuadrados.

Para la planificación correcta del trabajo debe realizarse un cálculo muy aproximado de los materiales necesarios en la ejecución del proyecto. En la preparación del molde flexible se considera suficiente un espesor de 2 centímetros. Como se trabaja por etapas, formando el molde por la conjunción de diversos módulos que permiten mantenerse en el "tiempo óptimo de trabajo", debe contemplarse aproximadamente un consumo de masilla del $10 \%$ por encima de la estrictamente necesaria para la superficie calculada. En este proyecto se precisan 20 litros de elastómero por cada metro cuadrado de superficie. De lo cual de deduce un gasto de 360 litros para la superficie y 36 litros para el solapamiento de los módulos. El consumo total aproximado de elastómero de silicona para el molde flexible es, pues, de 396 litros.

En la preparación del soporte rígido, el espesor del soporte adecuado es de 3 centímetros. Cada metro de superficie a cubrir requiere 30 litros de masilla de poliéster. Para construir un sistema modular hay que añadir un $10 \%$ mas de la masilla total de superficie. Con ello obtenemos los siguientes números: 540 litros de masilla de poliéster y 54 litros para el solapamiento de módulos. Esto hace un total de 594 litros de consumo de masilla de poliéster.

Debe tenerse en cuenta que el grosor del molde y del soporte puede ser conveniente variarlos en función de las circunstancias y según diversos criterios. En otros casos deberán recalcularse las cantidades de material necesario.

Una vez a punto los productos necesarios, se procede a la preparación de la masilla de silicona para obtener el molde flexible. Para ello se mezclan los materiales antes citados, en las proporciones señaladas, rellenando recipiente de 5 litros correspondientes a las tareas de aplicación según el "tiempo óptimo de trabajo". 

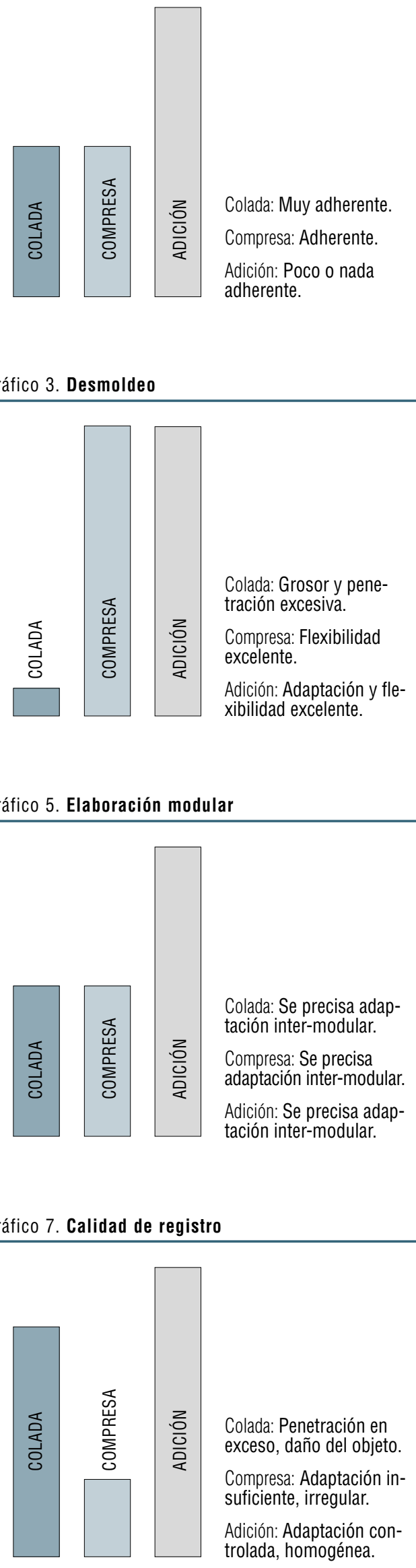

A continuación se sumerge el operador con el recipiente procediendo a la aplicación manual de su contenido sobre unos 25 centímetros cuadrados de superficie. El operador controla el grosor y la distribución del producto. No debe tardarse mas de 40 minutos en la aplicación de un recipiente.

La superficie de los módulos no corresponde necesariamente a la obtenida con el volumen de un recipiente de preparación. El elastómero "mordiente" adhiere bien sobre el "curado" pudiendo aumentar la superficie de un módulo a voluntad.

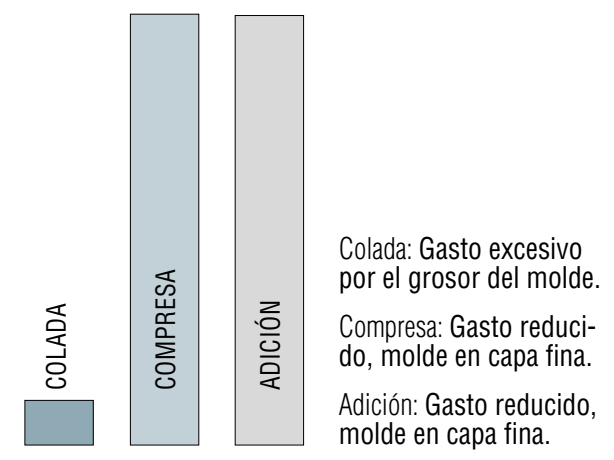
trolada, homogénea. 


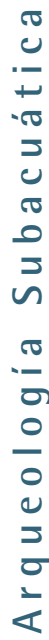

\section{Productos y marcas comerciales}

Base Silastic 34I8I de DOW CORNING®. Especificaciones técnicas aportadas por el fabricante-DOW CORNING. PRODUCT SAFETY DATA SHEET, 30/08/94-: caracterización técnica = elastómero de silicona, nombre = silicato de zirconio, color = blanco, viscosidad $=4000$ máximo mPa.s, RTV $=7,10$ r.p.m. después de 8 minutos, cambio de viscosidad $=20$ al
$60 \%$, peso específico del producto preparado en hoja de 2 milímetros y curado durante 7 días a $22^{\circ} \mathrm{C}$, $50 \%$ de humedad relativa $=1,17-1,23$, dureza SHORE $A=16-26$, resistencia a la rotura, $\mathrm{mPa}=$ 3,7 mínimo, alargamiento a la rotura, \% = 400 mínimo, resistencia al desgarro, $\mathrm{KN} / \mathrm{m}=18$ mínimo.

Agente de curado Silastic 8I de DOW CORNING®. Especificaciones técnicas aportadas por el fabricante -DOW CORNING. PRODUCT SAFETY DATA SHEET, 0I/07/94-: caracterización química = compuesto orgánico, nombre $=$ tetraetil silicato, forma $=$ líquido, color $=$ transparente, peso específico $=0,95$, viscosi$\mathrm{dad}=30 \mathrm{mPa}$, volatilidad $=<40 \%$.

Aditivo tixotrópico Silastic de DOW CORNING®. Especificaciones técnicas aportadas por el fabricante -DOW CORNING. PRODUCT SAFETY DATA SHEET, 12/07/94-: caracterización química = silicona, for$\mathrm{ma}=$ líquido, color $=$ ambar, viscosidad $=310 \mathrm{cst}$, peso específico $=1,04$, volatilidad $=10 \%$.

Resina de poliéster RESIPOL tixotrópica (BH-7|9). Especificaciones técnicas generales : nombre $=$ poli $(\mathrm{I}-$ fenil-etileno), temperatura de vitrificación $=80^{\circ} \mathrm{C}$, índice de refracción a temperatura ambiente (20-25 $\left.{ }^{\circ} \mathrm{C}\right)=1,59$, módulo de elasticidad $=3500$, estabilidad $=C(20$ años -6 meses $)$.

Sílice coloidal pirogenada LO-VEL 27. Especificaciones técnicas: nombre = bióxido de silicio puro ( $\mathrm{SiO} 2)$, presentación = polvo blanco finísimo, superficie $=$ $150 \mathrm{~m} 2 /$ gr., tamaño de partícula primaria = 21 nanómetros, $\mathrm{pH}=6,5$ a $25^{\circ} \mathrm{C}$.

\section{Notas}

I. MARTINEZ, B.; TOLEDO, E.: Moldes de silicona para piezas de madera procedentes de barcos sumergidos. "Pátina", Vol. 8, 1993, pp. 183-188.

2. FLORIAN, Mary-Lou E.: Scope and History of Archaeological Wood. Advances in Chemistry Series. Archaeological Wood: properties, chemistry and preservation. Washington, 1990, pp. 3-34

3. CRONYN, J.M.: The Elements of Archaeological Conservation $4^{\mathrm{a}}$ ed. London: Routledge, 1990, 360 pp. 0-415-0I207-4. pp. 57.

4. Se citan los trabajos mas relevantes: MURDOCK, L.D.; DALEY, T.: Progress report on the use of FMC polysulfide rubber compounds for recording archaeological ships' features in a marine environment. "The International Journal of Nautical Archaeology and Underwater Exploration", Vol. II, n 4, 1982, pp. 349-353.

MURDOCK, L.D.; DALEY, T.: Polysulfide rubber and its application for recording archaeological ship features in a marine envi- ronment. "The International Journal of Nautical Archaeology and Underwater Exploration", Vol. 10, n 4, 1981, pp. 337-342. MARTINEZ, B.; TOLEDO, E.: Moldes de silicona para piezas de madera procedentes de barcos sumergidos. "Pátina", Vol. 8 , 1993, pp. 183-188.

5. ZAMBRANO, Luis Carlos. Nuevo procedimiento para la obtención de moldes subacuáticos. Inventor: el mismo solicitante. ES-2 II 9 649. España. Patente de invención. 950।565. 1995.08 .95$.

6. op. cit.: MURDOCK, L.D.; DALEY, T (198I) ref. 4

7. DAVID, R.; DESCLAUX, M.: Le moulage subaquatique. Pour copie conforme. Paris: Serre, 1988. pp. 315-316.

8. op. cit.: DAVID, R.; DESCLAUX, M. (1988) ref. 6

9. HORIE, C.V:: Materials for Conservation. Organic consolidants, adhesives and coatings. $2^{\text {a }}$ ed.. Kent: Butterworths, 1987, 281 pp. 0-408-0।53।-4. pp. 183. 